Published in final edited form as:

Cancer Nurs. 2017 ; 40(3): 174-183. doi:10.1097/NCC.0000000000000405.

\title{
Persistent Fatigue in Hematopoietic Stem Cell Transplantation Survivors
}

\author{
Eileen Danaher Hacker, PhD, Anne M. Fink, PhD, Tara Peters, BS, Chang Park, PhD, \\ Giamila Fantuzzi, PhD, and Damiano Rondelli, MD \\ Department of Biobehavioral Health Science, College of Nursing, University of Illinois at Chicago \\ (Drs. Hacker and Fink, Ms. Peters); Department of Health Science Systems, College of Nursing, \\ University of Illinois at Chicago (Dr. Park): Department of Kinesiology and Nutrition, College of \\ Applied Health Sciences, University of Illinois at Chicago (Dr. Fantuzzi): Department of \\ Hematology/Oncology, College of Medicine, University of Illinois at Chicago (Dr. Rondelli).
}

\section{Abstract}

Background-Fatigue is highly prevalent following hematopoietic stem cell transplantation (HCT). It has been described as intense and may last for years following treatment.

Objective-to compare fatigue, physical activity, sleep, emotional distress, cognitive function, and biological measures in HCT survivors with persistent fatigue $(n=25)$ to age- and gendermatched healthy controls with occasional tiredness $(n=25)$.

Methods-Data were collected using: (a) objective, real-time assessments of physical activity and sleep over 7 days; (b) patient-reported fatigue assessments; (c) computerized objective testing of cognitive functioning; and (d) biological measures. Differences between groups were examined using MANOVA.

Results-HCT survivors reported increased physical $(p<.001)$, mental $(p<.001)$, and overall fatigue $(p<.001)$ as well as increased anxiety $(p<.05)$ and depression $(p<.01)$ compared to healthy controls. Red blood cell (RBC) levels were significantly lower in HCT survivors $(p<$. $001)$. RBC levels for both groups, however, were in the normal range. TNF- $a(p<.001)$ and IL-6 $(p<.05)$ were significantly higher in HCT survivors.

Conclusions-Persistent fatigue in HCT survivors compared to healthy controls with occasional tiredness is accompanied by increased anxiety and depression along with decreased RBCs. Elevated TNF- $a$ and IL- 6 may be important biomarkers.

Implications for Practice-This study provides preliminary support for the conceptualization of fatigue as existing on a continuum, with tiredness anchoring one end and exhaustion the other. Persistent fatigue experienced by HCT survivors is more severe than the occasional tiredness of everyday life.

Corresponding Author: Eileen Hacker, PhD, University of Illinois at Chicago, Department of Biobehavioral Health Science, 845 S. Damen Ave., (M/C 802), Room 760, Chicago, IL 60612, 312-996-7924, FAX 312-996-4979, ehacker@uic.edu.

Disclosures

There are no conflicts of interest. 


\section{Keywords}

Fatigue; cancer survivors; stem cell transplantation; anxiety; depression

\section{Introduction}

Understanding persistent fatigue in hematopoietic stem cell transplantation (HCT) survivors is crucial for expanding symptom science, particularly as it relates to long-term cancer survivorship. According to recent estimates, over 20,000 transplants are performed annually in the United States, ${ }^{1}$ and these numbers are expected to increase. The treatment is not without risk. HCT and the preparatory high-dose chemotherapy regimens given prior to HCT are associated with significant mortality ${ }^{1}$ and long-term morbidity, including persistent fatigue..$^{2,3}$

Fatigue is defined as a subjective lack of physical and/or mental energy, despite adequate access to sleep or rest periods, which is perceived by individuals to be out of proportion to recent activity and interferes with usual and desired activities over time. ${ }^{4}$ The prevalence and severity of fatigue immediately following HCT has been described as intense, with some cancer survivors reporting problems years following treatment. ${ }^{3,5} \mathrm{Up}$ to $44 \%$ of long-term HCT survivors report persistent moderate to severe fatigue even though they are disease free. ${ }^{2,6-10}$ In other cancer survivors, persistent fatigue is frequently associated with decreased physical activity, ${ }^{11,12}$ sleep disturbances, ${ }^{13,14}$ emotional distress, ${ }^{15}$ and cognitive dysfunction. ${ }^{16}$ Biological markers associated with fatigue and the stress response include elevated cytokines, particularly interleukin-6 (IL-6). ${ }^{17}$ In HCT patients, increases in IL-6 and soluble tumor necrosis factor (TNF)-R1 significantly predicted increasing severity of symptoms, including fatigue, during the acute post-transplant period. ${ }^{18}$ Little is known, however, about the association between fatigue and biomarkers in long-term HCT survivors with persistent fatigue.

As the number of HCT survivors continues to grow, symptoms or problems that impact long-term outcomes need to be identified. No studies found in the literature comprehensively examined persistent fatigue and the relationships among persistent fatigue, physical activity, sleep disturbances, emotional distress, cognitive function, and inflammatory biomarkers in HCT survivors. Unlike fatigue associated with cancer and active cancer treatment, persistent fatigue in HCT survivors may be a distinct and separate phenomenon that more closely resembles, chronic fatigue syndrome now known as systemic exertion intolerance disease than the fatigue associated with cancer and active cancer treatment. ${ }^{19}$ Most of the work examining persistent fatigue in cancer survivors has been conducted in breast cancer survivors ${ }^{20,21}$ or heterogeneous groups of cancer survivors. ${ }^{22}$ In HCT survivors, it is unknown how persistent fatigue compares to occasional tiredness normally experienced as part of everyday life and why some HCT survivors continue to experience fatigue in the absence of cancer and cancer treatment. Systematic characterization of persistent fatigue, physical activity, sleep, emotional distress, and cognitive function as experienced by HCT survivors compared to a group of matched healthy controls with occasional tiredness would facilitate our understanding of fatigue as a biobehavioral phenomenon. From a cancer 
survivor perspective, providing information regarding the difference between occasional tiredness and persistent fatigue lasting more than six months is critical for helping HCT survivors navigate through their new normal. This pilot study examined fatigue, physical activity, sleep, emotional distress, cognitive functioning, and biological measures, including inflammatory biomarkers, in HCT survivors with persistent fatigue lasting more than six months compared to age- and gender-matched healthy controls with occasional tiredness.

\section{Conceptual Framework}

Understanding the conceptual differences among tiredness, fatigue, and exhaustion is a difficult problem facing researchers who are interested in studying fatigue in cancer survivors, such as those who have undergone HCT. Fatigue and tiredness are frequently interchanged in the lay and health care literature. This creates conceptual confusion when trying to compare persistent fatigue following HCT to occasional tiredness that is normally experienced as part of everyday life. The Fatigue Adaptation Model suggests that tiredness, fatigue, and exhaustion represent three distinct behavioral states that exist on an adaptation continuum, with tiredness anchoring one end and exhaustion the other.participants ${ }^{23}$ Applying Selye's General Adaptation Syndrome, tiredness occurs during the alarm stage, fatigue occurs during the resistance phase and, when the body resources become depleted, exhaustion (the final stage) ensues. ${ }^{24}$ Tiredness precedes fatigue and fatigue precedes exhaustion. Differences exist in key behavioral domains as one moves from tiredness to fatigue to exhaustion.

The Fatigue Adaptation Model was used as an organizing framework and guided selection of potential biobehavioral correlates of persistent fatigue. ${ }^{23}$ According to the model, behavioral correlates of tiredness include feeling sleepy, impatient, absentminded, gradual loss of energy consistent with energy expended, and muscle weakness following work. With adequate rest, tiredness resolves, and the adaptive response is successful. In this study, we hypothesized that healthy people who have experienced occasional tiredness during the past seven days are able to adapt through rest; thus, preventing the progression from tiredness to fatigue. In HCT survivors with persistent fatigue lasting more than six months, the adaptation has not been successful and the behavioral correlates associated with fatigue and possibly even exhaustion may be present. The working hypothesis is HCT survivors with persistent fatigue experience decreased physical activity, more sleep disturbances or not feeling rested despite adequate sleep, emotional distress, cognitive dysfunction and altered biological markers typically associated with fatigue.

\section{Methods}

\section{Design}

This pilot study used a comparative descriptive design to evaluate fatigue, physical activity, sleep, emotional distress, cognitive functioning, and inflammatory biomarkers in HCT survivors with persistent fatigue that is not relieved with rest compared to age- and gendermatched healthy controls with occasional tiredness that resolves with rest. To capture patient-reported and objectively measured data, a combination of methodological approaches were used: (1) patient-reported assessments of fatigue using a standard self- 
report questionnaire (Chalder Fatigue Scale) and computerized Adult Short Forms (fatigue, sleep disturbances, and emotional distress) of the Patient Reported Outcomes Measurement Information System (PROMIS); (2) objective, real-time assessments of physical activity and sleep over seven days using wrist actigraphy; (3) computerized objective testing of cognitive functioning; and (4) biological measures (complete blood count [CBC]/Differential; creactive protein [CRP]; TNF- $a$; and IL-6).

\section{Sample}

The sample $(N=50)$ was composed of adult HCT survivors with persistent fatigue ( $n=25)$ and matched healthy controls with occasional tiredness $(n=25)$. The HCT population was specifically selected as the preparatory high-dose chemotherapy regimens given prior to HCT are prime examples of intensive cancer therapy. The more aggressive or intensive the cancer therapy, the more likely one is to develop persistent fatigue; ${ }^{25-28}$ thus, the HCT population serves as an excellent model for conducting a biobehavioral examination of persistent fatigue in cancer survivors.

Eligibility criteria were established for all participants with additional criteria for the healthy controls and HCT groups. All information was self-reported. Eligibility criteria for both groups included: (1) at least 18 years of age; (2) able to ambulate without assistance; (3) no history of major psychiatric disorder; (4) no current major anxiety or depressive disorder; and, (5) no current diagnosis of a sleep disorder, such as sleep apnea. Healthy controls met the following additional inclusion criteria: (1) self-reported occasional tiredness in the past seven days; (2) tiredness generally relieved with rest; (3) no history of a chronic condition, such as diabetes, heart disease, cancer, etc.; and, (4) no history of a condition in which fatigue is a common complication, such as multiple sclerosis. Healthy control subjects were excluded if they had any infectious, autoimmune/inflammatory diseases or were taking immunosuppressive medications. The healthy controls were matched by gender and age (within 5 years) to the HCT survivors.

All HCT survivors met the following inclusion criteria: (1) complete remission of cancer for at least one year post allogeneic or autologous HCT; (2) self-reported persistent fatigue during the previous 6 months; and, (3) fatigue generally not relieved with rest. HCT survivors with severe acute graft-versus-host disease (Grade III and IV), extensive chronic graft-versus-host disease, and/or taking steroids for another medical condition were excluded from the study.

The HCT survivors with persistent fatigue were recruited through local flyers, web advertisements and postings on BMT Info Network (http://www.bmtinfonet.org/). Normal healthy controls were recruited though word of mouth, local board and internet postings as well as ResearchMatch, an online registry for research volunteers.

\section{Instrumentation}

Major depression and anxiety disorders-All potential participants were screened for major depression and anxiety disorders using the Hospital Anxiety and Depression Scale (HADS) during recruitment. ${ }^{29}$ The HADS is a 14 -item instrument rated on a 4-point Likert 
scale; reliability and validity are well-established.$^{30}$ Item scores are summed to form a subscale score, with higher scores indicating higher levels of depression or anxiety. Designed for outpatients with medical illnesses, and widely used in people with chronic disease, somatic indicators of emotional distress or items referring to a clinical cause are excluded. This reduces bias toward symptoms resulting from concurrent medical conditions. Potential participants were initially screened with the HADS. Potential participants with subscale scores $\geq 19$, indicating major anxiety or depression disorders, were ineligible for study participation.

Fatigue-Fatigue was measured with the Chalder Fatigue Scale ${ }^{31}$ and the Fatigue Short Form (PROMIS instrument). ${ }^{32}$ The Chalder Fatigue Scale is a self-report instrument that measures physical and mental fatigue. The 11-item instrument has excellent psychometric properties and has been successfully used in community-dwelling participants. ${ }^{33,34}$ A 4point Likert scale for responses is employed. Higher scores indicate greater fatigue. Normative mean scores for the overall fatigue score have been reported as 12.2 (S.D. $=4.0$ ) in the general Norwegian population. ${ }^{35}$ The Adult Short Forms of the PROMIS instruments, including fatigue, are described in detail below.

Physical activity and sleep-Physical activity and sleep were objectively measured using a wrist-worn accelerometer (Actiwatch-Score, Philips Respironics, Bend, OR). This omnidirectional accelerometer monitors the occurrence, degree, and magnitude of physical activity (PA). The piezoelectric motion sensor integrates degree and speed of motion and stores the information as activity counts in one-minute epochs on the Actiwatch-Score ${ }^{\circledR}$ memory. PA is reported as the average PA count per minute over seven days. Intra- and interinstrument reliability for the wrist-worn accelerometer were established using a shaker table under six different conditions of various intensity. ${ }^{36}$ Intra-instrument and inter-instrument reliability has been established, as well as concurrent validity in people with chronic illnesses. ${ }^{37}$ Wrist actigraphy has been successfully used in HCT patients, with expected reductions in physical activity immediately following HCT providing further support for convergent validity. ${ }^{38}$

Sleep variables were computed using Actiware® software (V. 6.0). Data are stored in one minute epochs and the software scores each epoch as wake or sleep. Using an automated process, the software determines sleep start by searching for the first 10 minutes during which no more than one epoch is scored as "wake." The software assigns rest, sleep, or active intervals based on activity counts. The default software settings were used for the analysis, as recommended by the manufacturer. The sleep parameters produced include total sleep time, sleep latency (minutes until sleep onset), sleep efficiency, frequency and duration of awakenings after sleep onset (WASO), and number of awakenings. Sleep variables were scored for each 24-hour period, and a mean was computed for nighttime sleep. Intra and inter-instrument reliability was established using a shaker table under six different conditions of various intensity to produce a range of accelerometry counts. ${ }^{36}$ Wrist actigraphy is highly correlated with polysomnography, the gold standard for assessing sleep. ${ }^{39}$ Compared to polysomnography, wrist actigraphy is highly accurate (86\%) and 
sensitive (96\%) for detecting sleep. ${ }^{40}$ It has been successfully used to assess sleep and sleep disturbances across a wide range of populations, including people with cancer. ${ }^{38,41}$

Fatigue, sleep disturbance, and emotional distress-The Adult Short Forms of PROMIS were used to measure fatigue, ${ }^{32}$ sleep disturbances, ${ }^{42}$ and emotional distress (anger, anxiety, and depression). ${ }^{43}$ These instruments are reliable, valid, and precise. ${ }^{44,45}$ Computerized versions of the five short forms (fatigue, sleep disturbances, anger, anxiety, and depression) were used in this study. The PROMIS instruments use a 5-point Likert scale and employ 7-day recall. Scores are standardized $(M=50, S D=10)$ and normed to a reference (U.S.) population. Higher scores indicate higher levels of fatigue, sleep disturbances, and emotional distress (anger, anxiety, and depression).

Cognitive functioning was assessed using well-established and objective neuropsychological tests. ${ }^{46}$ Computerized versions of tests (CNS Vital Signs Clinical Core Battery, Morrisville, NC) that have established psychometric properties with available normative data were used. ${ }^{47}$ The specific tests included: (1) Verbal Memory for memory recognition for words; (2) Visual Memory for memory recognition for figures; (3) Finger Tapping Test for motor speed and visual-motor ability; (4) Symbol Digit Coding for psychomotor speed; (5) Stroop Test for information processing speed; (6) Shifting Attention Test for executive function; and (7) Continuous Performance Test for sustained attention.

Biological measures-Venous blood samples were obtained from an antecubital arm vein and drawn into heparinized tubes for cytokine and CRP determination and into tubes containing ethylenediaminetetraacetic acid for the CBC/Differential. Venipuncture was selected as the method for blood collection considering reports of increased local cytokine production in response to an indwelling catheter. ${ }^{48}$ All participants provided samples during the day time, and the technicians documented the exact time of blood collection considering reports that a subject's morning and afternoon values could differ. ${ }^{49}$ Ninety two percent of samples $(n=45)$ were drawn before 1:00 pm. To obtain plasma for cytokine and CRP analysis, tubes were centrifuged at $13,200 \mathrm{rpm}$ at $4^{\circ} \mathrm{C}$ for $10 \mathrm{~min}$. Plasma was separated into aliquots of $200 \mu \mathrm{l}$ each and frozen at $-80^{\circ} \mathrm{C}$. Cytokine determination for local participants was determined using the Quantikine ${ }^{\circledR}$ high-sensitivity enzyme-linked immunoassay (R\&D Systems, Minneapolis, MN). Assays from non-local participants were performed by Quest Diagnostic Laboratories ${ }^{\circledR}$ using high-sensitivity enzyme-linked immunoassay. CRP was determined by end point nephelometry, and CBC/Differential was performed using automated microscopy and colorimetric method (Quest Diagnostic Laboratories®).

\section{Data Collection Schedule and Procedures}

The institutional review board approved this study. Potential participants responded to recruitment flyers and web advertisements for research. Participants self-identified as HCT survivors with persistent fatigue or healthy controls with occasional tiredness. A telephone screening script was used to determine eligibility. All screening information was selfreported. Potential participants who were eligible and expressed interest in participating in the study were mailed informed consent documents. Upon receipt of the signed written informed consent document, a wrist actigraph was shipped to participants. Participants were 
contacted by telephone to ascertain arrival and confirm placement on the nondominant arm. Participants wore the devices continuously for seven days. At the end of the seven-day period, participants removed the wrist actigraphs and completed the Chalder Fatigue Scale, PROMIS instruments, and cognitive function tests. Blood samples were drawn at this time. All healthy controls and most HCT survivors $(n=10)$ made one visit to the site to complete questionnaires, cognitive functioning tests, and blood draws. Non-local HCT participants ( $n$ $=15$ ) completed questionnaires and cognitive function testing in their home with a member of the research staff available via telephone. Biological samples were drawn at a national diagnostic laboratory near the non-local HCT participants' home.

\section{Data Analysis}

Descriptive statistics were computed for all variables to ensure the quality of the data (check distributions, examine outliers) and to describe the sample. Because multiple dependent variables were believed to be conceptually related, multivariate analysis of variance (MANOVA) was used to evaluate differences between the HCT survivors with persistent fatigue and healthy controls with occasional tiredness. All MANOVAs were conducted with subject group as the independent variable. The MANOVA models examined fatigue, anxiety/ depression, cognitive functioning, objective physical activity/sleep, and all PROMIS instruments. Body mass index (BMI) is a potential confounding variable for IL-6 and CRP. ${ }^{50}$ For this reason, BMI was controlled statistically using MANCOVA with BMI entered as a co-variate to examine the biological measures. We were primarily interested in identifying trends in data as this was a pilot study. An alpha level of .05 was used for all statistical tests.

\section{Results}

\section{Demographic and Treatment Characteristics}

The mean age of the sample was $52.8(S D=11.8)$ with more males $(n=28,56 \%)$ than females $(n=22,44 \%)$. Age $\left(M_{\mathrm{HCT}}\right.$ survivor $\left.=53.4, S D=11.5 ; M_{\mathrm{HC}}=52.3, S D=12.2\right)$ and gender were comparable among groups. The sample was predominantly White $(n=42)$, followed by Black/African American $(n=3)$, Hispanic $(n=1)$, Asian $(n=1)$, and unreported/unknown $(n=3)$. The demographic characteristics are reported in Table 1 and self-reported clinical characteristics for the HCT survivors are described in Table 2. Descriptive statistics for all other variables are reported in Table 3. Allogeneic HCT recipients constituted $76 \%$ of the HCT with persistent fatigue group ( $n=19$; sibling, matched unrelated, and haploidentical donors). There were no significant differences between autologous and allogeneic recipients on any of the variables.

\section{Fatigue}

The MANOVA with overall, physical, and mental fatigue (Chalder Fatigue Scale) and fatigue score from the Fatigue PROMIS score as dependent variables indicated significant differences between the groups: Pillai's Trace $=.576, \mathrm{~F}_{(3,45)}=20.340, p<.001$ (Figure). Follow-up univariate ANOVAs revealed significant group differences for overall fatigue (Chalder Fatigue Scale), $\mathrm{F}_{(1,47)}=52.701, p<.001$; physical fatigue, $\mathrm{F}_{(1,47)}=37.527, p<$. 001 ; mental fatigue, $\mathrm{F}_{(1,47)}=47.171, p<.001$; and, fatigue (PROMIS), $\mathrm{F}_{(1,47)}=22.646, p$ 
$<.001$. HCT survivors reported significantly more overall, physical, and mental fatigue than healthy controls with occasional tiredness, thus, clearly differentiating the two groups.

\section{Anxiety and Depression}

The MANOVA with anxiety (anxiety subscale of the HADS and anxiety PROMIS) and depression (depression subscale of the HADS and depression PROMIS) as dependent variables indicated significant differences between the groups: Pillai's Trace $=.294, \mathrm{~F}_{(4,44)}=$ 4.586, $p<.005$. Follow-up univariate ANOVAs revealed significant group differences for anxiety (HADs subscale), $\mathrm{F}_{(1,47)}=52.701, p<.001$; anxiety (PROMIS), $\mathrm{F}_{(1,47)}=5.122, p$ $<.05$; depression (HADS subscale), $\mathrm{F}_{(1,47)}=15.159, p<.001$; and depression (PROMIS), $\mathrm{F}_{(1,47)}=7.426, p<.01$. Therefore, HCT survivors with persistent fatigue reported significantly more anxiety and depression than healthy controls with occasional tiredness.

\section{Physical Activity and Sleep}

The MANOVA with physical activity, total sleep time, sleep latency, sleep efficiency, frequency and duration of awakenings after sleep onset, and number of awakenings as dependent variables indicated no significant differences between groups.

\section{Cognitive Functioning}

The MANOVA with composite memory, verbal memory, visual memory, psychomotor speed, reaction time, complex attention, cognitive flexibility, processing speed, and executive function as dependent variables indicated no significant differences between groups. The single self-report item, "How is your memory?" from the Chalder Fatigue Scale was analyzed separately. HCT participants with persistent fatigue self-reported significantly worse memory compared to healthy controls with occasional tiredness; $\mathrm{t}_{(47)}=7.678, \mathrm{p}<$. 001 .

\section{Biological Measures}

The MANCOVA with WBC, RBC, hemoglobin, hematocrit, CRP, IL-6 and TNF- $\alpha$ as dependent variables indicated significant differences between the groups: Pillai's Trace $=$. $486, \mathrm{~F}_{(7,36)}=4.869, p=.001$. Follow-up univariate ANOVAs revealed significant group differences for RBCs, $\mathrm{F}_{(1,42)}=15.842, p<.001$; IL-6, $\mathrm{F}_{(1,42)}=5.458, \mathrm{p}<.05$; and TNF- $\mathrm{a}$, $\mathrm{F}_{(1,42)}=11.803, p=.001$. Though RBC levels were significantly lower in HCT survivors with persistent fatigue compared to healthy controls with occasional tiredness, the mean levels for both groups were in the normal range. IL- 6 and TNF- $a$ levels were significantly higher in HCT survivors with persistent fatigue compared to healthy controls with occasional tiredness. There was no correlation between IL-6 or TNF- $a$ and time of day for blood draw.

\section{Discussion}

Moderate to severe persistent fatigue affects almost half of HCT survivors, yet little is known how persistent fatigue in this population differs from occasional tiredness found in the general population. This study provides preliminary evidence for the biobehavioral correlates of persistent fatigue lasting more than six months in HCT survivors who are 
cancer free. Compared to healthy controls with occasional tiredness, HCT survivors with persistent fatigue experience greater anxiety and depression along with along with decreased RBC and hemoglobin levels. TNF- $a$ and IL-6 levels were significantly elevated in the HCT survivors compared to the healthy controls and this may represent an important mechanistic pathway for fatigue via chronic inflammation. The roles of physical activity, sleep, and cognitive functioning, however, are less clear. These findings suggests a clear distinction between fatigue and tiredness and identifies accompanying symptoms and biological differences. These findings provide clear direction for future examination of persistent fatigue and its potential biobehavioral correlates and may have important implications for interventions designed to reduce fatigue in long-term HCT survivors.

As expected, there were large significant differences between the HCT survivors and healthy controls on all fatigue measures, indicating that the persistent fatigue experienced by HCT survivors is conceptually and empirically different than the occasional tiredness experienced by healthy controls. Multiple methodological strategies were implemented in this study to strengthen the design and, ultimately, confidence in the findings. For example, age- and gender-matched healthy controls with occasional tiredness were specifically chosen as the comparison group as it was imperative to first demonstrate that fatigue could be differentiated from tiredness when using common fatigue instruments that employ a 7-day recall. Potential participants with major depressive, anxiety, and/or sleep disorders were screened out in both groups reducing the likelihood of fatigue from other conditions. In addition, participants in the healthy control group were free from disease normally associated with fatigue further reducing this bias. Moreover, fatigue was measured using two different, well-established instruments increasing the confidence in the results. Additional methodological strategies in future studies might include the addition of a third group, HCT participants with occasional tiredness. A three-group study (HCT survivors with persistent fatigue, HCT survivors with occasional tiredness, and healthy controls with occasional tiredness) would explicate the differences between persistent fatigue in HCT survivors and occasional tiredness in HCT survivors as well as healthy controls further advancing our understanding of fatigue and cancer symptom science.

Advancing symptom science requires interpretation of study findings within the context of previous research. The study builds upon the work by Gielissen et al. who reported that $35-41 \%$ of long-term HCT survivors $(n=98)$ experienced severe fatigue one to fifteen years post-transplant. ${ }^{2}$ In the Gielissen study, dysregulated physical activity and sleep disturbances, using patient-reported measures, predicted severe fatigue. Building upon these findings, the current study included an age- and gender-matched control group and measured variables using objective assessments in addition to self-report. The findings from our study suggest that fatigue persists in HCT survivors compared to healthy controls despite adequate access to rest and sleep. There were no significant differences between HCT survivors and controls on any objectively measured sleep parameters or self-report of sleep disturbances. These findings were somewhat surprising given the strong link between sleep and fatigue. On the other hand, these findings are consistent with the definition of cancer-related fatigue: fatigue despite adequate access to rest and sleep. Confidence in the findings are strengthened by the concurrent use of patient-reported sleep disturbances and objective sleep assessments 
obtained over seven days. Our results, however, only provide preliminary evidence and must be interpreted with caution due to the small sample size.

Emotional distress, a known biobehavioral correlate of fatigue, may be particularly problematic for some long-term HCT survivors and contribute to persistent fatigue in this population. Numerous psychological and cognitive sequelae have been documented following HCT, such as anxiety and depression, ${ }^{51,52}$ cognitive dysfunction, ${ }^{53}$ and memory deficits memory deficits. ${ }^{54}$ In the current study, anxiety and depression were significantly higher in HCT survivors compared to the healthy controls. Conversely, no significant differences were found between the two groups on any of the objective cognitive function tests despite the fact that the HCT survivors in this study reported significantly worse memory. The reason for the inconsistency between self-reported memory problems and objective memory tests is not known, and requires further examination.

Understanding the underlying mechanistic pathways to fatigue at the cellular level are important for advancing symptom science. In this study, HCT survivor group had significantly lower RBC levels. While anemia is associated with fatigue, the RBC and hemoglobin levels in the HCT survivors were within normal range. Importantly, the oxygen carrying capacity as measured by hemoglobin was not statistically different between the two groups; yet, the HCT survivors reported significantly greater fatigue. This demonstrates the physiological complexity and persistence of fatigue even in the presence of normal laboratory values for oxygen carrying capacity. IL-6 and TNF-a levels were higher among HCT survivors compared to controls. These findings are consistent with findings in breast cancer survivors with persistent fatigue and suggest chronic inflammation as a possible mechanistic pathway. ${ }^{55}$ This is tempered by the finding that differences in CRP levels were expected but not found between the HCT survivors and healthy controls. Methodological procedures and the small sample size may account for the inability to find significant differences for CRP levels. The pathobiological/behavioral pathways to persistent fatigue have not been clearly articulated and this represents an important direction for future research, particularly as it relates to developing targeted interventions.

Addressing the breadth and depth of the fatigue assessment and its associated biobehavioral correlates has important research and clinical implications. One of the most pressing difficulties associated with conducting fatigue research in HCT survivors is deciding upon the conceptual and operational definitions of fatigue and determining which, if any, biobehavioral correlates to build into the research. There is no universally accepted measure of fatigue in people with cancer and this leads to the inability to generalize across studies. The sheer number of biobehavioral correlates of fatigue and the complexity of the relationships tests our ability to identify and include a reasonable number of biobehavioral variables in research studies to make a meaningful and comprehensive contribution without overwhelming participants. Barsevick and colleagues introduced an organizing framework for cancer-related fatigue that identifies demographic, medical, behavioral/well-being, functional and biological factors associated with fatigue representing an important step for determining the most salient factors to assess in any given research study. ${ }^{56}$ The organizing framework also provides an important tool for clinicians. Conducting a thorough assessment of fatigue and potential contributing factors is crucial for developing a clinical management 
strategy. The Survivorship: Fatigue, V. 1.2014 (Clinical Practice Guidelines in Oncology) ${ }^{57}$ and the Oncology Nursing Society's fatigue PEP guidelines ${ }^{58}$ provide clear and realistic guidance for assessing and managing fatigue in clinical practice settings.

Finally, several limitations potentially limit the generalizability of this study's findings. Other variables not assessed in this pilot study may impact the development of persistent fatigue in HCT survivors, such as type of conditioning regimen. For example, allogeneic HCT patients receiving a myeloablative compared to reduced intensity conditioning regimen may be at higher risk for developing persistent fatigue. Other treatment and/or clinical variables in HCT survivors, such as length of time since diagnosis and/or other comorbid conditions not assessed in this study, may also influence the development of fatigue. The clinical characteristics of all participants, including the HCT survivors, were self-reported in this study. Performing a complete health history or abstracting clinical and treatment data from medical records in future studies would provide greater insight into these relationships. Lastly, all of the participants in this study self-identified as either HCT survivors with persistent fatigue lasting more than six months or healthy controls with occasional tiredness. This sampling technique may have introduced selection bias as neither group may be representative of their respective populations.

\section{Conclusion}

This pilot study provides preliminary conceptual and empirical support for the conceptualization of fatigue as existing on a continuum, with tiredness anchoring one end and exhaustion the other. The findings demonstrate that fatigue experienced by HCT survivors is quantitatively distinct from occasional tiredness experienced by age- and gender-matched healthy controls. HCT patients with persistent fatigue reported greater anxiety and depression and experienced reduced RBC levels (albeit in the normal range) and elevated TNF- $a$ and IL-6 levels compared to healthy controls. Although HCT survivors reported impaired memory, the objective cognitive function tests were comparable to the controls. Finally, this study provides preliminary evidence that other symptoms and biological markers accompany persistent fatigue. Importantly, future research should include a variety of methodological approaches to assess fatigue and other biobehavioral factors to adequately capture the breadth and depth of this symptom to advance our understanding in HCT survivors.

\section{Acknowledgments}

This study was supported in part by the National Cancer Institute, Alliance for Clinical Trials in Oncology grant, previously the Cancer and Leukemia Group B through Grant CA037447 (E. Hacker, PI) and the National Center for Advancing Translational Sciences, National Institutes of Health, through Grant UL1TR000050. The content is solely the responsibility of the authors and does not necessarily represent the official views of the National Institutes of Health. The authors thank Kevin Grandfield, Publication Manager of the UIC Department of Biobehavioral Health Science, for editorial assistance.

\section{REFERENCES}

1. Pasquini, MC., Wang, Z. [May 5, 2015] Current use and outcome of hematopoietic stem cell transplantation: 2014 CIBMTR Summary Slides. http://www.cibmtr.org. 
2. Gielissen MF, Schattenberg AV, Verhagen CA, Rinkes MJ, Bremmers ME, Bleijenberg G. Experience of severe fatigue in long-term survivors of stem cell transplantation. Bone Marrow Transplant. 2007; 39(10):595-603. [PubMed: 17369868]

3. Grulke N, Albani C, Bailer H. Quality of life in patients before and after haematopoietic stem cell transplantation measured with the European Organization for Research and Treatment of Cancer (EORTC) Quality of Life Core Questionnaire QLQ-C30. Bone Marrow Transplant. 2012; 47(4): 473-482. [PubMed: 21602898]

4. Berger AM, Abernethy AP, Atkinson A, et al. Cancer-related fatigue. J. Natl. Compr. Canc. Netw. 2010; 8(8):904-931. [PubMed: 20870636]

5. Bishop MM, Beaumont JL, Hahn EA, et al. Late effects of cancer and hematopoietic stem-cell transplantation on spouses or partners compared with survivors and survivor-matched controls. J. Clin. Oncol. 2007; 25(11):1403-1411. [PubMed: 17416860]

6. Conner-Spady BL, Cumming C, Nabholtz JM, Jacobs P, Stewart D. A longitudinal prospective study of health-related quality of life in breast cancer patients following high-dose chemotherapy with autologous blood stem cell transplantation. Bone Marrow Transplant. 2005; 36(3):251-259. [PubMed: 15937502]

7. Mosher CE, Redd WH, Rini CM, Burkhalter JE, Duhamel KN. Physical, psychological, and social sequelae following hematopoietic stem cell transplantation: a review of the literature. Psychooncology. 2008

8. Andrykowski MA, Carpenter JS, Greiner CB, et al. Energy level and sleep quality following bone marrow transplantation. Bone Marrow Transplant. 1997; 20(8):669-679. [PubMed: 9383231]

9. Andrykowski MA, Bishop MM, Hahn EA, et al. Long-term health-related quality of life, growth, and spiritual well-being after hematopoietic stem-cell transplantation. J. Clin. Oncol. 2005; 23(3): 599-608. [PubMed: 15659507]

10. Hjermstad MJ, Knobel H, Brinch L, et al. A prospective study of health-related quality of life, fatigue, anxiety and depression 3-5 years after stem cell transplantation. Bone Marrow Transplant. 2004; 34(3):257-266. [PubMed: 15170167]

11. Lynch BM, Cerin E, Newman B, Owen N. Physical activity, activity change, and their correlates in a population-based sample of colorectal cancer survivors. Ann. Behav. Med. 2007; 34(2):135-143. [PubMed: 17927552]

12. Stevinson C, Steed H, Faught W, et al. Physical activity in ovarian cancer survivors: associations with fatigue, sleep, and psychosocial functioning. Int. J. Gynecol. Cancer. 2009; 19(1):73-78. [PubMed: 19258945]

13. Alexander S, Minton O, Andrews P, Stone P. A comparison of the characteristics of disease-free breast cancer survivors with or without cancer-related fatigue syndrome. Eur. J. Cancer. 2008

14. Bower JE, Ganz PA, Irwin MR, Kwan L, Breen EC, Cole SW. Inflammation and behavioral symptoms after breast cancer treatment: do fatigue, depression, and sleep disturbance share a common underlying mechanism? J. Clin. Oncol. 2011; 29(26):3517-3522. [PubMed: 21825266]

15. Duijts SF, van Egmond MP, Spelten E, van Muijen P, Anema JR, van der Beek AJ. Physical and psychosocial problems in cancer survivors beyond return to work: a systematic review. Psychooncology. 2014; 23(5):481-492. [PubMed: 24375630]

16. Jacobs SR, Jacobsen PB, Booth-Jones M, Wagner LI, Anasetti C. Evaluation of the functional assessment of cancer therapy cognitive scale with hematopoietic stem cell transplant patients. J. Pain Symptom Manage. 2007; 33(1):13-23. [PubMed: 17196903]

17. Bower JE. Cancer-related fatigue: links with inflammation in cancer patients and survivors. Brain. Behav. Immun. 2007; 21(7):863-871. [PubMed: 17543499]

18. Wang XS, Shi Q, Williams LA, et al. Serum interleukin-6 predicts the development of multiple symptoms at nadir of allogeneic hematopoietic stem cell transplantation. Cancer. 2008; 113(8): 2102-2109. [PubMed: 18792065]

19. Servaes P, van der Werf S, Prins J, Verhagen S, Bleijenberg G. Fatigue in disease-free cancer patients compared with fatigue in patients with chronic fatigue syndrome. Support Care Cancer. 2001; 9(1):11-17. [PubMed: 11147137] 
20. Alexander S, Minton O, Andrews P, Stone P. A comparison of the characteristics of disease-free breast cancer survivors with or without cancer-related fatigue syndrome. Eur. J. Cancer. 2009; 45(3):384-392. [PubMed: 18977131]

21. Bower JE. Behavioral symptoms in patients with breast cancer and survivors. J. Clin. Oncol. 2008; 26(5):768-777. [PubMed: 18258985]

22. Goedendorp MM, Gielissen MF, Verhagen CA, Bleijenberg G. Development of fatigue in cancer survivors: a prospective follow-up study from diagnosis into the year after treatment. J. Pain Symptom Manage. 2013; 45(2):213-222. [PubMed: 22926087]

23. Olson K. A new way of thinking about fatigue: a reconceptualization. Oncol Nurs Forum. 2007; 34(1):93-99. [PubMed: 17562637]

24. Selye, H. The story of the adaptation syndrome. Acta; Montreal, CA: 1952.

25. Servaes P, Verhagen S, Schreuder HW, Veth RP, Bleijenberg G. Fatigue after treatment for malignant and benign bone and soft tissue tumors. J. Pain Symptom Manage. 2003; 26(6):11131122. [PubMed: 14654263]

26. de Jong N, Candel MJ, Schouten HC, Abu-Saad HH, Courtens AM. Prevalence and course of fatigue in breast cancer patients receiving adjuvant chemotherapy. Ann. Oncol. 2004; 15(6):896905. [PubMed: 15151946]

27. Bower JE, Ganz PA, Desmond KA, et al. Fatigue in long-term breast carcinoma survivors: a longitudinal investigation. Cancer. 2006; 106(4):751-758. [PubMed: 16400678]

28. Woo B, Dibble SL, Piper BF, Keating SB, Weiss MC. Differences in fatigue by treatment methods in women with breast cancer. Oncol. Nurs. Forum. 1998; 25(5):915-920. [PubMed: 9644708]

29. Zigmond AS, Snaith RP. The hospital anxiety and depression scale. Acta Psychiatr. Scand. 1983; 67(6):361-370. [PubMed: 6880820]

30. DeWalt DA, Rothrock N, Yount S, Stone AA, Group. PC. Evaluation of item candidates: The PROMIS qualitative review. Medical Care. 2007; 45(5):S12-S21. [PubMed: 17443114]

31. Chalder T, Berelowitz G, Pawlikowska T, et al. Development of a fatigue scale. J. Psychosom. Res. 1993; 37(2):147-153. [PubMed: 8463991]

32. Garcia SF, Cella D, Clauser SB, et al. Standardizing patient-reported outcomes assessment in cancer clinical trials: a patient-reported outcomes measurement information system initiative. J. Clin. Oncol. 2007; 25(32):5106-5112. [PubMed: 17991929]

33. Loge JH, Abrahamsen AF, Ekeberg O, Kaasa S. Hodgkin's disease survivors more fatigued than the general population. J Clin Oncol. 1999; 17(1):253-261. [PubMed: 10458240]

34. Cella M, Chalder T. Measuring fatigue in clinical and community settings. J. Psychosom. Res. 2010; 69(1):17-22. [PubMed: 20630259]

35. Loge JH, Ekeberg O, Kaasa S. Fatigue in the general Norwegian population: normative data and associations. J. Psychosom. Res. 1998; 45(1):53-65. [PubMed: 9720855]

36. Esliger DW, Tremblay MS. Technical reliability assessment of three accelerometer models in a mechanical setup. Medicine and science in sports and exercise. 2006; 38(12):2173-2181. [PubMed: 17146326]

37. Steele BG, Holt L, Belza B, Ferris S, Lakshminaryan S, Buchner DM. Quantitating physical activity in COPD using a triaxial accelerometer. Chest. 2000; 117(5):1359-1367. [PubMed: 10807823]

38. Hacker ED, Ferrans C, Verlen E, et al. Fatigue and physical activity in patients undergoing hematopoietic stem cell transplant. Oncol Nurs Forum. 2006; 33(3):614-624. [PubMed: 16676017]

39. Ancoli-Israel S, Cole R, Alessi C, Chambers M, Moorcroft W, Pollak CP. The role of actigraphy in the study of sleep and circadian rhythms. Sleep. 2003; 26(3):342-392. [PubMed: 12749557]

40. Marino M, Li Y, Rueschman MN, et al. Measuring sleep: accuracy, sensitivity, and specificity of wrist actigraphy compared to polysomnography. Sleep. 2013; 36(11):1747-1755. [PubMed: 24179309]

41. Dean GE, Abu Sabbah E, Yingrengreung S, et al. Sleeping with the enemy: sleep and quality of life in patients with lung cancer. Cancer Nurs. 2015; 38(1):60-70. [PubMed: 25486204] 
42. Buysse DJ, Yu L, Moul DE, et al. Development and validation of patient-reported outcome measures for sleep disturbance and sleep-related impairments. Sleep. 2010; 33(6):781-792. [PubMed: 20550019]

43. Pilkonis PA, Choi SW, Reise SP, et al. Item banks for measuring emotional distress from the Patient-Reported Outcomes Measurement Information System (PROMIS(R)): depression, anxiety, and anger. Assessment. 2011; 18(3):263-283. [PubMed: 21697139]

44. Cella D, Riley W, Stone A, et al. The Patient-Reported Outcomes Measurement Information System (PROMIS) developed and tested its first wave of adult self-reported health outcome item banks: 2005-2008. J. Clin. Epidemiol. 2010; 63(11):1179-1194. [PubMed: 20685078]

45. Rothrock NE, Hays RD, Spritzer K, Yount SE, Riley W, Cella D. Relative to the general US population, chronic diseases are associated with poorer health-related quality of life as measured by the Patient-Reported Outcomes Measurement Information System (PROMIS). J. Clin. Epidemiol. 2010; 63(11):1195-1204. [PubMed: 20688471]

46. Michiels V, Cluydts R. Neuropsychological functioning in chronic fatigue syndrome: a review. Acta. Psychiatr. Scand. 2001; 103(2):84-93. [PubMed: 11167310]

47. Gualtieri CT, Johnson LG. Reliability and validity of a computerized neurocognitive test battery, CNS Vital Signs. Arch Clin Neuropsychol. 2006; 21(7):623-643. [PubMed: 17014981]

48. Gudmundsson A, Ershler WB, Goodman B, Lent SJ, Barczi S, Carnes M. Serum concentrations of interleukin-6 are increased when sampled through an indwelling venous catheter. Clin. Chem. 1997; 43(11):2199-2201. [PubMed: 9365415]

49. Altara R, Manca M, Hermans KC, et al. Diurnal rhythms of serum and plasma cytokine profiles in healthy elderly individuals assessed using membrane based multiplexed immunoassay. J. Transl. Med. 2015; 13:129. [PubMed: 25903806]

50. Fink AM, Gonzalez RC, Lisowski T, et al. Fatigue, inflammation, and projected mortality in heart failure. J. Card. Fail. 2012; 18(9):711-716. [PubMed: 22939040]

51. Lee SJ, Loberiza FR, Antin JH, et al. Routine screening for psychosocial distress following hematopoietic stem cell transplantation. Bone Marrow Transplant. 2005; 35(1):77-83. [PubMed: 15502851]

52. Chang G, Orav EJ, McNamara TK, Tong MY, Antin JH. Psychosocial function after hematopoietic stem cell transplantation. Psychosomatics. 2005; 46(1):34-40. [PubMed: 15765819]

53. Harder H, Cornelissen JJ, Van Gool AR, Duivenvoorden HJ, Eijkenboom WM, van den Bent MJ. Cognitive functioning and quality of life in long-term adult survivors of bone marrow transplantation. Cancer. 2002; 95(1):183-192. [PubMed: 12115332]

54. Rusiewicz A, DuHamel KN, Burkhalter J, et al. Psychological distress in long-term survivors of hematopoietic stem cell transplantation. Psychooncology. 2008; 17(4):329-337. [PubMed: 17621377]

55. Bower JE, Ganz PA, Irwin MR, Castellon S, Arevalo J, Cole SW. Cytokine genetic variations and fatigue among patients with breast cancer. J. Clin. Oncol. 2013; 31(13):1656-1661. [PubMed: 23530106]

56. Barsevick AM, Irwin MR, Hinds P, et al. Recommendations for high-priority research on cancerrelated fatigue in children and adults. J. Natl. Cancer Inst. 2013; 105(19):1432-1440. [PubMed: 24047960]

57. Denlinger CS, Ligibel JA, Are M, et al. Survivorship: fatigue, version 1.2014. J. Natl. Compr. Canc. Netw. 2014; 12(6):876-887. [PubMed: 24925198]

58. Mitchell SA, Hoffman AJ, Clark JC, et al. Putting evidence into practice: an update of evidencebased interventions for cancer-related fatigue during and following treatment. Clin. J. Oncol. Nurs. 2014; 18(Suppl):38-58. [PubMed: 25427608] 


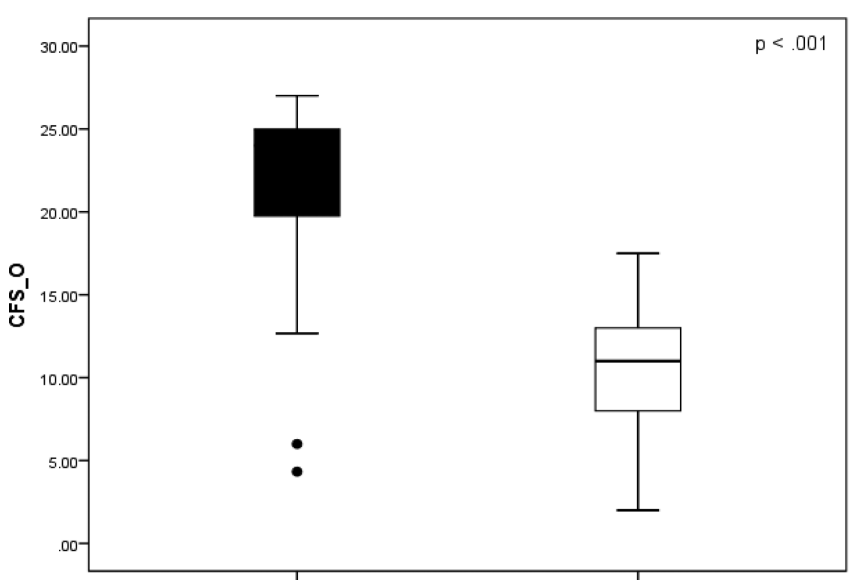

HCT Survivors

Healthy Controls

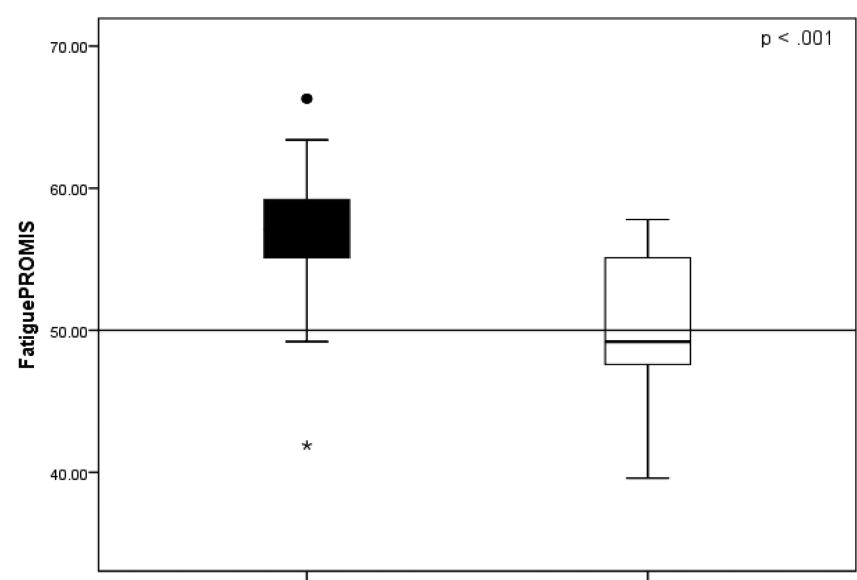

HCT Survivors

Healthy Controls

\section{Figure.}

Fatigue (Overall Fatigue-Chalder Fatigue Scale and Fatigue PROMIS): Median, 25\% and $75 \%$. 


\section{Table 1}

Demographic Characteristics $(\mathrm{N}=50)$

\begin{tabular}{|c|c|c|c|}
\hline \multirow[b]{2}{*}{ Characteristics } & \multicolumn{3}{|c|}{ Value } \\
\hline & HCT Survivors $(n=25)$ & Healthy Controls $(\mathrm{n}=\mathbf{2 5})$ & Overall $(\mathbf{N}=\mathbf{5 0})$ \\
\hline Age, M (SD) & $53.4(11.5)$ & $52.3(12.2)$ & $52.8(11.8)$ \\
\hline \multicolumn{4}{|l|}{ Gender, N (\%) } \\
\hline Male & $14(56)$ & $14(56)$ & $28(56)$ \\
\hline Female & $11(44)$ & $11(44)$ & $22(44)$ \\
\hline \multicolumn{4}{|l|}{ Race, N (\%) } \\
\hline African American & & $3(12)$ & $3(6)$ \\
\hline Asian & & $1(4)$ & $1(2)$ \\
\hline Hispanic & & $1(4)$ & $1(2)$ \\
\hline White & $22(88)$ & $20(80)$ & $42(84)$ \\
\hline Not Reported & $3(12)$ & & $3(6)$ \\
\hline \multicolumn{4}{|l|}{ Education, $\mathrm{N}(\%)$} \\
\hline Graduated from High & $1(4)$ & $1(4)$ & $2(4)$ \\
\hline \multicolumn{4}{|l|}{ School } \\
\hline Some College & $4(16)$ & $9(36)$ & $13(26)$ \\
\hline Graduated from College & $10(40)$ & $6(24)$ & $16(32)$ \\
\hline Graduated Education after & $8(32)$ & $9(36)$ & $17(34)$ \\
\hline \multicolumn{4}{|l|}{ College } \\
\hline Not Reported & $2(8)$ & & $2(4)$ \\
\hline
\end{tabular}

Cancer Nurs. Author manuscript; available in PMC 2018 May 01. 
Table 2

Clinical Characteristics of the HCT Survivors $(n=25)$

\begin{tabular}{ll}
\hline Characteristics & Value \\
\hline Months from Transplant, M (SD) & $63(52)$ \\
Months from Transplant, Median (Range) & $43(13-238)$ \\
Diagnosis, N (\%) & \\
Acute myelogenous/lymphoblastic leukemia & $8(32)$ \\
Chronic myelogenous leukemia & $2(8)$ \\
Myelodysplastic syndrome & $2(8)$ \\
Lymphoma & $7(28)$ \\
Multiple myeloma & $3(12)$ \\
Other & $3(12)$ \\
Donor type, N (\%) & \\
Autologous & $6(24)$ \\
Sibling & $13(52)$ \\
Matched Donor & $4(16)$ \\
Haploidentical & $2(8)$ \\
\hline
\end{tabular}


Table 3

Patient-Reported and Objective Assessments of Variables.

\begin{tabular}{|c|c|c|c|}
\hline Variable & HCT Survivors M (SD) & Healthy Controls M (SD) & $P$ Value \\
\hline \multicolumn{4}{|l|}{ Fatigue } \\
\hline Overall (Chalder Fatigue Scale) & $21.28(6.07)$ & $10.39(4.31)$ & $p<.001$ \\
\hline Physical (Chalder Fatigue Scale) & $13.36(4.30$ & $6.67(3.31)$ & $p<.001$ \\
\hline Mental (Chalder Fatigue Scale) & $7.92(2.52)$ & $3.72(1.70)$ & $p<.001$ \\
\hline Fatigue (PROMIS) & $57.04(5.12)$ & $50.48(4.52)$ & $p<.001$ \\
\hline \multicolumn{4}{|l|}{ Anxiety } \\
\hline Anxiety (HADS) & $6.16(3.31)$ & $4.08(2.31)$ & $p<.001$ \\
\hline Anxiety (PROMIS) & $59.33(7.88)$ & $54.46(7.19)$ & $p<.05$ \\
\hline \multicolumn{4}{|l|}{ Depression } \\
\hline Depression (HADS) & $6.04(3.58)$ & $2.80(1.96)$ & $p<.001$ \\
\hline Depression (PROMIS) & $54.12(8.49)$ & $47.08(9.52)$ & $p<.01$ \\
\hline Anger (PROMIS) & $53.45(8.59)$ & $49.27(9.57)$ & $n s$ \\
\hline Sleep Disturbances (PROMIS) & $54.79(3.49)$ & $54.04(3.24)$ & $n s$ \\
\hline Physical Activity (activity counts per min) & $240.51(74.32)$ & $259.63(72.41)$ & $n s$ \\
\hline \multicolumn{4}{|l|}{ Objective Sleep Assessments } \\
\hline Total Sleep Time (min) & $422.48(79.60)$ & 378.08 (53.18) & $n s$ \\
\hline Sleep Latency (min) & $11.70(9.59)$ & $15.90(18.50)$ & $n s$ \\
\hline Sleep Efficiency (percent) & $83.41(4.85)$ & $81.59(7.79)$ & $n s$ \\
\hline Wake after Sleep Onset (min) & $60.24(19.28)$ & $57.11(22.26)$ & $n s$ \\
\hline Number of Awakenings & $26.85(6.82)$ & $23.28(5.87)$ & $n s$ \\
\hline \multicolumn{4}{|l|}{ Cognitive Functioning } \\
\hline Composite Memory (percent) & $51.00(22.67)$ & $50.44(25.25)$ & $n s$ \\
\hline Verbal Memory (percent) & $35.71(21.12)$ & $43.20(33.97)$ & $n s$ \\
\hline Visual Memory (percent) & $65.42(23.62)$ & $59.28(27.95)$ & $n s$ \\
\hline Psychomotor Speed (percent) & $42.04(25.85)$ & $53.48(24.95)$ & $n s$ \\
\hline Reaction Time (percent) & $32.63(24.67)$ & $47.80(29.93)$ & $n s$ \\
\hline Complex Attention (percent) & $46.29(31.02)$ & $48.20(26.45)$ & $n s$ \\
\hline Cognitive Flexibility (percent) & $39.83(28.73)$ & $48.00(30.87)$ & $n s$ \\
\hline Processing Speed (percent) & $48.25(28.95)$ & $53.68(23.65)$ & $n s$ \\
\hline Executive Function (percent) & $43.08(28.33)$ & $48.68(30.56)$ & $n s$ \\
\hline \multicolumn{4}{|l|}{ Biological measures } \\
\hline RBC (Million/uL) & $4.17(0.44)$ & $4.72(0.43)$ & $p<.001$ \\
\hline Hemoglobin $(\mathrm{g} / \mathrm{dL})$ & $13.46(1.30)$ & $14.16(1.26)$ & $n s$ \\
\hline Hemocrit (percent) & $40.40(4.07$ & $42.33(3.61)$ & $n s$ \\
\hline $\mathrm{CRP}(\mathrm{mg} / \mathrm{dL})$ & $0.21(0.24)$ & $0.25(0.41)$ & $n s$ \\
\hline IL-6 (pg/mL) & $2.34(2.06)$ & $1.56(1.11)$ & $p<.05$ \\
\hline TNF-a $(\mathrm{pg} / \mathrm{mL})$ & $1.85(0.66)$ & $1.13(0.70)$ & $p=.001$ \\
\hline
\end{tabular}

ns: No significant differences between HCTsurvivors and healthy controls. 